\title{
VO and GPS integrated navigation algorithm based on plane constraint
}

\author{
Chade $\mathrm{Lv}^{1,2}$, Qingxi Zeng ${ }^{1,2, *}$, Dehui Liu ${ }^{1}$, and Wenqi Qiu ${ }^{1}$ \\ ${ }^{1}$ College of Automation Engineering, Nanjing University of Aeronautics and Astronautics, Nanjing \\ 211106, China \\ ${ }^{2}$ Key Laboratory of Nuclear Technology Application and Radiation Protection in Astronautics \\ (Nanjing University of Aeronautics and Astronautics), Ministry of Industry and Information \\ Technology, Nanjing 211106, China
}

Keywords: Monocular VO, GPS, Integrated navigation, Adaptive Kalman filtering.

\begin{abstract}
In view of the deficiencies of GPS in the urban environment, such as signal occlusion, multipath and Non-LOS, this paper proposes a solution that using a low-cost monocular camera instead of inertial navigation system to combine with GPS. Firstly, considering that the actual road conditions of the vehicle are mostly plane and in order to reduce the computational resources of visual odometer (VO), this paper establishes a VO solving model based on plane constraint; then the VO\&GPS loose coupling model is proposed. The position error and yaw angle error of the two sensors are taken as input, and the errors are filtered by the adaptive Kalman filter. Experiments show that the positioning effect of VO\&GPS loose coupling system is better than that of single GPS, and it can suppress the drift of VO, which can meet the positioning requirements when satellite signals are interfered in a short time.
\end{abstract}

\section{Introduction}

Navigation positioning of vehicles is a key technology in the field of unmanned driving. Due to its own limitations, a single navigation and positioning system cannot meet the positioning accuracy requirements of unmanned vehicles, so integrated navigation technology is needed. At present, the INS/GPS integrated navigation system is the most common, but to obtain accurate vehicle positioning information, it is necessary to use the expensive INS to integrate with GPS, which will increase the vehicle cost. Therefore, a more suitable positioning scheme is needed to solve the above problems.

The development of computer vision has made vision-based positioning technology widely used [1] and visual odometer (VO) is gradually being used to assist GPS positioning. According to the classic GPS/INS loose combination system error model, a GPS/VO combination system is proposed in [2], which combines the data of both monocular VO and GPS through Kalman filter. The fusion algorithm proposed in [3] combines GPS carrier

*Corresponding author: autonavinuaa@163.com 
phase measurement with feature-based monocular visual odometer. The fusion algorithm can effectively suppress the error drift of the visual odometer even in the case that GPS can only receive two or three satellites. In [4], a EKF-based stereo vision and GPS fusion vehicle positioning method is proposed to reduce the cumulative error of stereo vision. A navigation system of the dual GPS fusion stereo camera is constructed in [5], which can provide vehicle status information position, attitude and speed for intelligent navigation. In [6], the Kalman filter is used to merge the binocular visual odometer positioning result with the GPS positioning result to improve the positioning accuracy of the integrated system. In [7], a method to improve the accuracy of GNSS positioning by binocular vision using the geographical location information of landmarks is proposed.

An algorithm for precise position based on low-cost camera combined with GPS is proposed in this paper. Considering that the actual road conditions of the vehicle are mostly planes and reducing the computational resources of $\mathrm{VO}$, this paper first establishes a VO solving model based on plane constraint. Then the VO\&GPS loose coupling model is proposed. The position error and yaw angle error of the two sensors are taken as input, and the error is filtered by the adaptive Kalman filter in the VO\&GPS model. Finally, the effectiveness of the algorithm is verified by experiments.

\section{Research on visual odometer based on plane constraint}

\subsection{Epipolar geometry constraint}

Epipolar geometry constraint is the key to the model of monocular vision odometer based on plane constraint. It describes the mapping relationship of a certain point in three-dimensional space between the perspective of two cameras. Where, the camera pose information reflected by the perspective of two cameras is included in the essential matrix $E$. Fig. 1 shows the basic principle of epipolar geometry constraint. The three-dimensional point $Q$ and its two-dimensional projection point $q_{1}$ and $q_{2}$ are coplanar under the camera perspective. If the camera perspective $C_{0}$ is fixed, it can be determined that the corresponding point of $q_{1}$ on the perspective image $C_{0}$ is located on the straight line $l_{2}$, which is called the polar line.In other words, the polar line $l_{2}$ is the epipolar line of the imaging point $q_{1}$, and there is also a epipolar line $l_{1}$ corresponding to $q_{2}$ on the imaging plane of the perspective $C_{0}$.

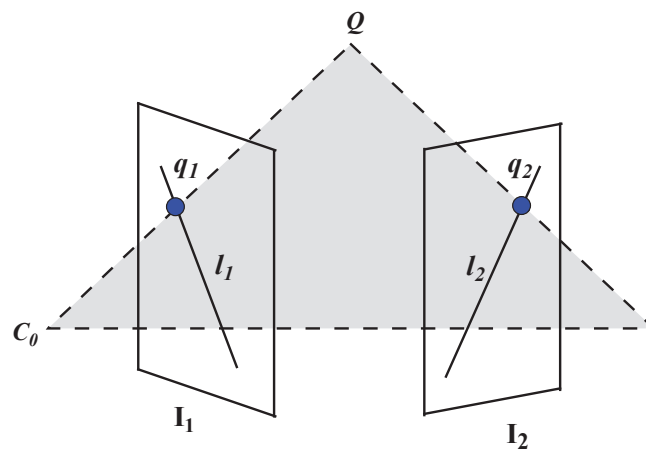

Fig. 1. Epipolar geometry model.

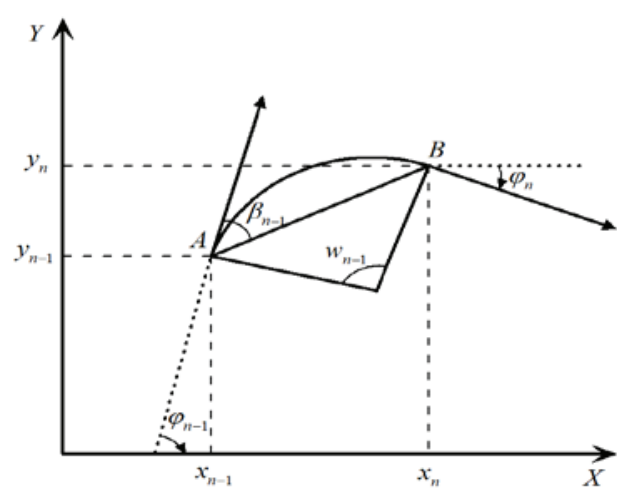

Fig. 2. Plane constrained VO solution model. 


\subsection{General 8-point pose solution}

According to the epipolar geometric constraint between two views:

$$
q_{1} F q_{2}{ }^{T}=0
$$

where, $F$ is the basic matrix, $q_{1}$ and $q_{2}$ are a pair of feature points in the image coordinate system. $q_{1}$ and $q_{2}$ are $1 * 3$ dimensional vectors, superscript $T$ represents the transposition of matrix. Order:

$$
q_{1}=(u, v, 1) \quad, \quad q_{2}=(x, y, 1) \quad, \quad F=\left[\begin{array}{lll}
f_{1} & f_{2} & f_{3} \\
f_{4} & f_{5} & f_{6} \\
f_{7} & f_{8} & f_{9}
\end{array}\right]
$$

Eq. 1 and Eq. 2 can be used to establish the linear homogeneous equation. The rank of coefficient matrix of the linear homogeneous equation is 8 . Only 8 pairs of matching feature points are needed to calculate the normalized basic matrix $F$. After that, calibrating the camera to estimate the internal parameter matrix $K$ of the camera. According to the basic matrix $F$ and the internal parameter matrix $K$ of the camera, the essential matrix $E$ can be obtained. The normalized rotation matrix $R$ and the shift matrix $T$ can be obtained by singular value decomposition of the essential matrix $E$.

The process of solving essential matrix $E$, rotation matrix $R$ and normalized translation matrix $T$ is as follows:

$$
E=K^{T} F K
$$

According to Eq. 3, the essential matrix $E$ can be obtained. The two non-zero singular values of the essential matrix $E$ are equal. Therefore, matrix $U$ and $V$ can be obtained by the following singular value decomposition method.

$$
E=\operatorname{Udiag}(1,1,0) V^{T}
$$

Through $U$ and $V$, four different groups of rotation matrix $R$ and translation matrix $T$ can be obtained. The calculation formula is shown in Eq. 5 .

$$
\begin{aligned}
& R=U W V^{T}, \quad T=U_{3} \\
& R=U W^{T} V^{T}, \quad T=U_{3} \\
& R=U W V^{T}, \quad T=-U_{3} \\
& R=U W^{T} V^{T}, \quad T=-U_{3}
\end{aligned} \quad, \quad W=\left[\begin{array}{ccc}
0 & -1 & 0 \\
1 & 0 & 0 \\
0 & 0 & 1
\end{array}\right]
$$

where $U_{3}$ represents the third row of the $U$ matrix. A pair of feature points are used to calculate the depth value of the point in two images. If both depth values are greater than 0 , the unique $R$ and $T$ are determined [8,9].

\subsection{Design of visual odometer algorithm based on plane constraint}

According to the knowledge of spatial rotation, the matrix $R_{n-1}^{n}$ can be obtained by rotating the vehicle coordinate system around the y-axis firstly in the n-time, and the rotation angle is defined as the yaw angle $w$,then rotating around the $\mathrm{x}$-axis, and the rotation angle is defined 
as the pitch angle $\gamma$; finally rotating around the z-axis, and the rotation angle is defined as the roll $\theta$. According to Eq. 5 and $R_{n-1}^{n}$, the variation of yaw angle $w_{n-1}$, pitch angle $\gamma_{n-1}$ and roll angle $\theta_{n-1}$ of the vehicle in the adjacent sampling interval can be obtained. The solution process is shown in Eq. 6.

$$
R_{n-1}^{n}(2,3)=\sin \gamma_{n-1}, \frac{R_{n-1}^{n}(2,1)}{R_{n-1}^{n}(2,2)}=\frac{\sin \theta_{n-1} \cos \gamma_{n-1}}{\cos \gamma_{n-1} \cos \theta_{n-1}}=\tan \theta_{n-1}, \frac{R_{n-1}^{n}(1,3)}{R_{n-1}^{n}(3,3)}=-\frac{\sin w_{n-1} \cos \gamma_{n-1}}{\cos w_{n-1} \cos \gamma_{n-1}}=-\tan w_{n-1}
$$

According to Eq. 6, the spatial coordinate system can be projected to the plane, and the vehicle can be simplified as a particle. As shown in Figure 2, the vehicle moves from point $A$ to point $B$ along the curve in the adjacent sampling interval, and the horizontal in-plane displacement $|\overrightarrow{A B}|$ of point $A$ to point $B$ can be measured through RTK (or single point GPS), and the yaw angle $w_{n-1}$ can be obtained through Eq.6. The centroid sideslip angle $\beta_{n-1}$ [10] can be expressed as the angle between the velocity of point $A$ and displacement $|\overrightarrow{A B}|$ in Fig. 1, and the calculation process is shown in Eq. 7.

$$
\frac{T(1)}{T(3)}=\tan \beta_{n-1}
$$

According to Fig. 2, the equation of position change and yaw angle change from point $A$ to point $B$ can be obtained, as shown in Eq. 8 .

$$
x_{n}=x_{n-1}+|\overrightarrow{A B}| \cos \left(\varphi_{n-1}-\beta_{n-1}\right) \quad, \quad y_{n}=y_{n-1}+|\overrightarrow{A B}| \sin \left(\varphi_{n-1}-\beta_{n-1}\right) \quad, \quad \varphi_{n}=\varphi_{n-1}+w_{n-1}
$$

\section{Performance analysis of visual odometer based on plane constraint}

\subsection{Acquisition of experimental data}

Since the monocular VO lacks the scale information, the $35 \mathrm{~m}$ long straight road was equidistantly marked at intervals of $10 \mathrm{~cm}$ before the experiment, and the separation distance was used as the scale of the monocular VO solution. The experiment was divided into three groups. The first group: the CCD camera was mounted on a tripod, as shown in Fig. 3(a). The pictures were taken continuously along the marked points of the $35 \mathrm{~m}$ long straight road, and the captured scene pictures are shown in Fig. 4. The second and third groups: the receiver was mounted on a tripod, as shown in Fig. 3(b), and the GPS and RTK data of the straight marker points were respectively collected. Where, the RTK data was used as the ground truth. Since the position of the RTK base station may not be very accurate, there may be some systematic error, but it does not affect the verification of the algorithm. The parameters of each experimental equipment are shown in Table 1. 
Table 1. Experimental equipment parameters

\begin{tabular}{ccccc}
\hline GPS frequency & RTK frequency & Camera frequency & Image size & Camera model \\
\hline $1 \mathrm{~Hz}$ & $5 \mathrm{~Hz}$ & $10 \mathrm{~Hz}$ & $800 * 600$ & CCD MER-125-30UM \\
\hline
\end{tabular}

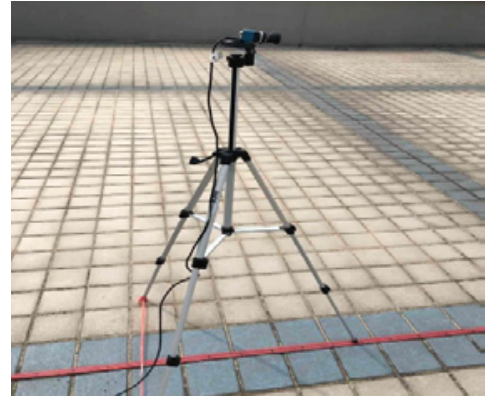

(a)

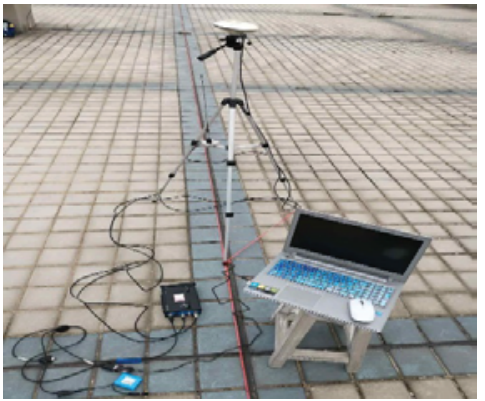

(b)

Fig. 3. Experimental platform construction.

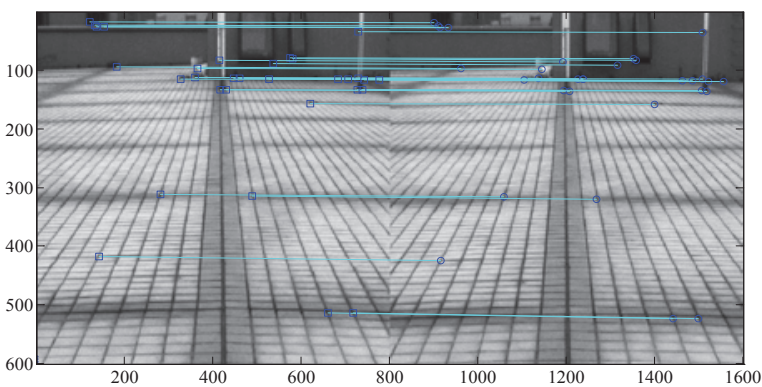

Fig.4. Matching effect of scene image.

\subsection{Monocular VO straight driving performance analysis}

The images collected by the experiment are solved according to the visual odometer model described in Section 2.3, and the calculated centroid sideslip angle change amount $\beta_{n-1}$ and yaw angle change amount $w_{n-1}$ are as shown in Fig. 5. During the straight running, the change of the centroid sideslip angle $\beta_{n-1}$ does not exceed $0.2^{\circ}$, and the change of yaw angle $w_{n-1}$ does not exceed $0.5^{\circ}$. The change of the centroid sideslip angle $\beta_{n-1}$ and the yaw angle $w_{n-1}$ in Fig. 4 are used to pose calculation by the Eq. 10. Where $|\overrightarrow{A B}|$ represents the scale of the monocular VO, and the specific value is the $10 \mathrm{~cm}$ equidistant interval of the straight road. After the solution, the trajectory of the monocular VO under the straight road is obtained, and the comparison of the monocular VO with the GPS and RTK trajectories is shown in Fig. 6. Before the $20 \mathrm{~m}$ of the straight road, the deviation of the VO trajectory is generally smaller than that of the GPS, which indicates that the monocular VO is more reliable than the single GPS positioning in a short time. Due to the cumulative error of the monocular VO, after $20 \mathrm{~m}$, the deviation of the VO trajectory begins to exceed the GPS. 


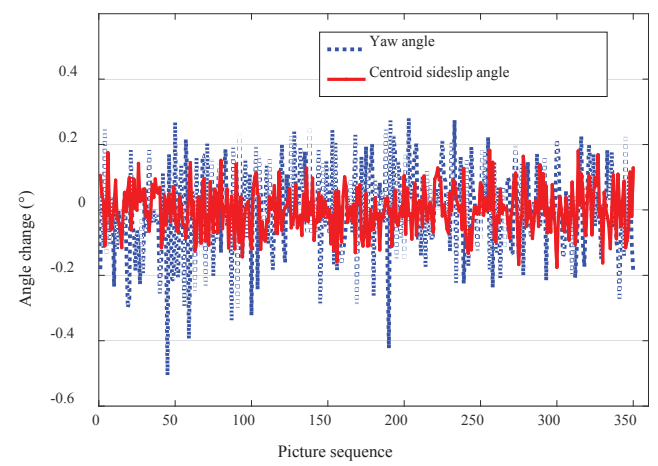

Fig. 5. Centroid sideslip angle and yaw angle change.

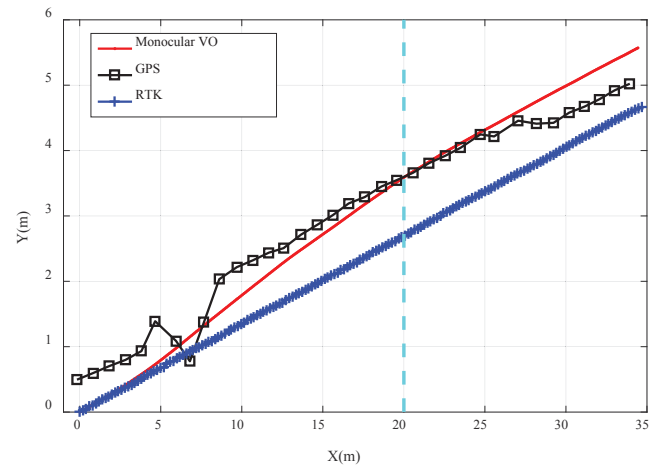

Fig. 6. Comparison of positioning trajectories.

\section{VO and GPS integrated navigation system research}

\subsection{VO\&GPS loose coupling system principle}

The integrated navigation system is divided into two modes: open-loop correction and closed-loop correction. This paper refers to the GPS and INS loose coupling system to build the open-loop correction system shown in Fig. 7. In practical engineering, the model error $Q$ and measurement error $R$ of the Kalman filter algorithm are difficult to be accurately measured. The accuracy of the estimation of $Q$ and $R$ determines the accuracy of the Kalman filter. Therefore, the adaptive Kalman filter [11] is used to fuse VO and GPS information.

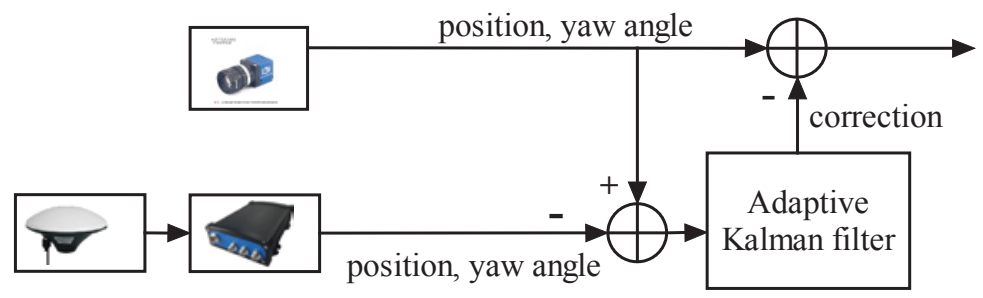

Fig. 7. Schematic diagram of the fusion system.

The state equation and measurement equation of the adaptive Kalman filter are shown in Eq. 9. 


$$
X_{k}=A X_{k-1}+w_{k-1} \quad, \quad Y_{k}=C X_{k}+v_{k-1}
$$

where, $\quad Y=\left[x_{v o}-x_{g p s}, y_{v o}-y_{g p s}, \varphi_{v o}-\varphi_{g p s}\right]^{T}, \quad X=[\Delta x, \Delta y, \Delta \varphi]^{T}, \quad A=\operatorname{diag}(1,1,1)$, $C=\operatorname{diag}(1,1,1)$. Both $w$ and $v$ are Gaussian white noise.

\subsection{VO\&GPS Loose Coupling System Straight Driving Performance Analysis}

The $10 \mathrm{~Hz}$ monocular VO data obtained by the solution and the interpolated GPS data are combined in accordance with the adaptive Kalman algorithm. The trajectory comparison between the VO\&GPS loose coupling system and other positioning systems is shown in Fig. 8. As can be seen from Fig. 8 , before the $20 \mathrm{~m}$ of the straight road, the trajectory of the VO\&GPS loose coupling system is significantly closer to the trajectory of the RTK than the GPS alone due to the assistance of VO. After the $20 \mathrm{~m}$ of the straight road, since the assistance of GPS weakens the influence of the cumulative error of $\mathrm{VO}$, the positioning error of the VO\&GPS loose coupling system is still in an acceptable range. The positioning errors of the three positioning systems in the $\mathrm{X}$ and $\mathrm{Y}$ directions are shown in Fig. 9 and Table 2, and they can also explain this problem. The above results can all indicate that the positioning effect of the VO\&GPS loose coupling system is better than that of the single GPS when running $35 \mathrm{~m}$ in a straight line, and it can suppress the cumulative drift of VO, which can meet the positioning requirements when the satellite signal is interfered in a short time.

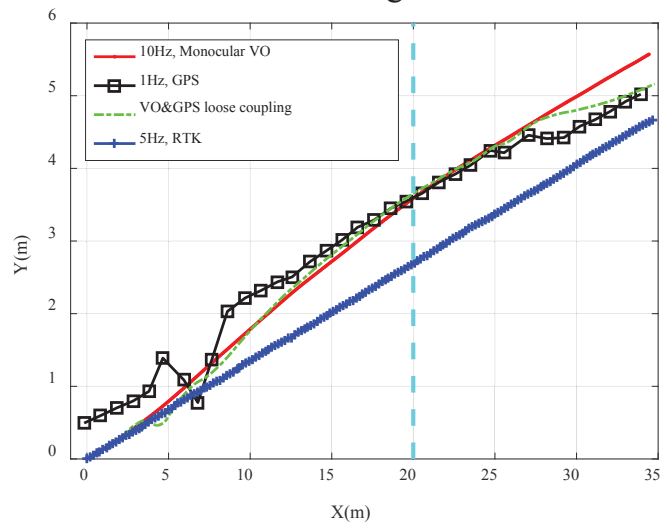

Fig. 8. Comparison of positioning trajectories.

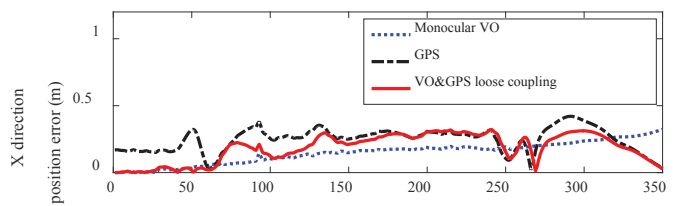

(a)

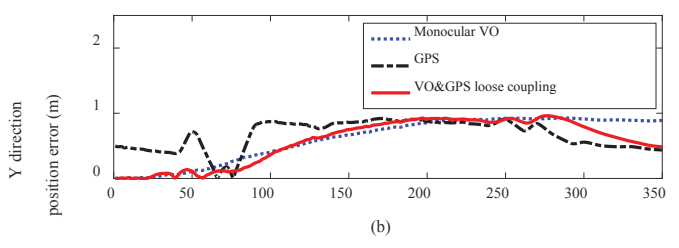

Time sequence

Fig. 9. Comparison of positioning error curves. 
Table 2. Positioning errors in X-Y directions.

\begin{tabular}{ccccc}
\hline Positioning System & $\begin{array}{c}\text { 0-20m average } \\
\text { error in X } \\
\text { direction }(\mathrm{m})\end{array}$ & $\begin{array}{c}\text { 0-20m average } \\
\text { error in Y } \\
\text { direction }(\mathrm{m})\end{array}$ & $\begin{array}{c}\text { 0-35m average } \\
\text { error in X } \\
\text { direction }(\mathrm{m})\end{array}$ & $\begin{array}{c}0-35 \mathrm{~m} \text { average } \\
\text { error in Y } \\
\text { direction }(\mathrm{m})\end{array}$ \\
\hline GPS & 0.2430 & 0.6569 & 0.2486 & 0.6671 \\
Monocular VO & 0.0970 & 0.4024 & 0.1492 & 0.6172 \\
VO\&GPS loose coupling & 0.1511 & 0.4059 & 0.1862 & 0.5754 \\
\hline
\end{tabular}

\section{Summary}

Firstly, this paper proposes a monocular VO solving model based on plane constraint. By taking offline processing of the pictures collected by the experiment, the solved monocular VO can still maintain high precision by running $35 \mathrm{~m}$ along the straight line. Then, the VO\&GPS loose coupling model is proposed. The position error and yaw angle error of the two sensors are taking as input, and the errors are filtered by an adaptive Kalman filter. When the VO\&GPS loose coupling system runs $35 \mathrm{~m}$ in a straight line, its average positioning error is better than the single GPS. The experimental results show that the VO\&GPS integrated navigation algorithm proposed in this paper can improve the positioning accuracy of the navigation system and suppress the cumulative drift of VO to meet the positioning requirements when satellite signals are interfered in a short time.

This research was financially supported by the Nanjing University of Aeronautics and Astronautics Graduate Innovation Base (Lab) Open Fund (kfjj20190312).

\section{References}

1. D. Scaramuzza, F. Fraundofer. "Visual Odometry Part I: The First 30 Years and Fundamentals." IEEE Robotics \& Automation Magazine, 2011, pp. 80-92.

2. D. Dusha, and L. Mejias. Error analysis and attitude observability of a monocular GPS/visual odometry integrated navigation filter. Sage Publications, Inc., 2012.

3. A. Soloviev, and D. Venable. "Integration of GPS and Vision Measurements for Navigation in GPS Challenged Environments." Position Location and Navigation Symposium (PLANS), 2010 IEEE/ION IEEE, 2010, pp. 1142-1149.

4. L. Wei, C. Cappelle, Y. Ruichek, et al. "Intelligent Vehicle Localization in Urban Environments Using EKF-based Visual Odometry and GPS Fusion." IFAC Proceedings Volumes, vol. 44, no. 1, pp. 13776-13781, 2011.

5. X. Jun. Research on the Key Technology of Intelligent Vehicle Navigation System based on Satellite and Vision. Wuhan: Wuhan University, 2011.

6. Z. Li. Road Detection and Obstacle Avoidance Technology Based on GNSS and Vision. Chengdu: University of Electronic Science and Technology, 2015.

7. Z. Yiran, G. Chengjun and N. Ruizhao. "Research on stereo-vision aided GNSS localization for intelligent vehicles." Computer Engineering and Applications, vol. 52, no. 17, pp. 192-197, 2016.

8. D. Scaramuzza, F. Fraundorfer and R. Siegwart. "Real-time monocular visual odometry for on-road vehicles with 1-point RANSAC." IEEE International Conference on Robotics \& Automation. IEEE Press, 2009, pp. 488-494.. 
9. D. Scaramuzza. "1-Point-RANSAC Structure from Motion for Vehicle-Mounted Cameras by Exploiting Non-holonomic Constraints." International Journal of Computer Vision, vol. 95, no. 1, pp. 74-85, 2011.

10. Z. Chenchen, X. Qunsheng and H. Le. "A Study on the Influence of Sideslip Angle at Mass Center on Vehicle Stability." Automotive Engineering, vol. 33, no. 4, pp. 277-282, 2011.

11. Y. Yulong. Research on Vector-based Tracking Algorithms for GNSS Signals. Harbin: Harbin Institute of Technology, 2012. 